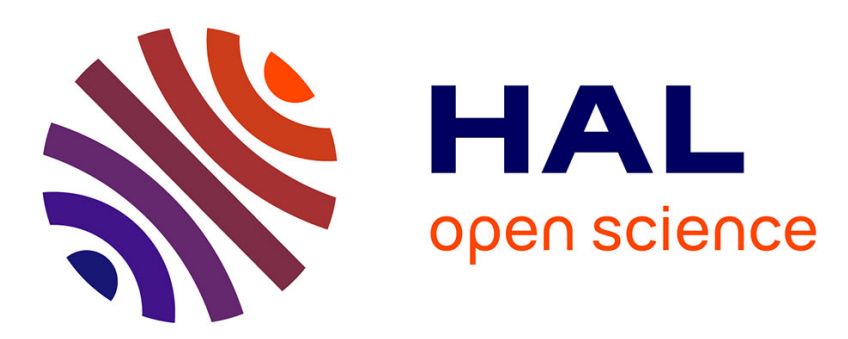

\title{
Denoising of ictal EEG data using semi-blind source separation methods based on time-frequency priors
}

Sepideh Hajipour, Mohammad Bagher, Laurent Albera, Isabelle Merlet

\section{To cite this version:}

Sepideh Hajipour, Mohammad Bagher, Laurent Albera, Isabelle Merlet. Denoising of ictal EEG data using semi-blind source separation methods based on time-frequency priors. IEEE Journal of Biomedical and Health Informatics, 2015, 19 (3), pp.839-847. 10.1109/JBHI.2014.2336797 . hal01246033

\section{HAL Id: hal-01246033 \\ https://hal.science/hal-01246033}

Submitted on 17 Dec 2015

HAL is a multi-disciplinary open access archive for the deposit and dissemination of scientific research documents, whether they are published or not. The documents may come from teaching and research institutions in France or abroad, or from public or private research centers.
L'archive ouverte pluridisciplinaire HAL, est destinée au dépôt et à la diffusion de documents scientifiques de niveau recherche, publiés ou non, émanant des établissements d'enseignement et de recherche français ou étrangers, des laboratoires publics ou privés. 


\title{
Denoising of ictal EEG data using semi-blind source separation methods based on time-frequency priors
}

\author{
Sepideh Hajipour Sardouie ${ }^{(1,2,3)}$, Student Member, IEEE, Mohammad Bagher Shamsollahi ${ }^{(3)}$, Senior \\ Member, IEEE, Laurent Albera ${ }^{(1,2,4)}$, Senior Member, IEEE, and Isabelle $\operatorname{Merlet}^{(1,2)}$ \\ (1) Inserm, UMR 1099, Rennes, F-35000, France \\ (2) LTSI, University of Rennes 1, Rennes, F-35000, France \\ (3) BiSIPL, Sharif University of Technology, Tehran, Iran \\ (4) INRIA, Centre Inria Rennes-Bretagne Atlantique, 35042 Rennes Cedex, France
}

\begin{abstract}
Removing muscle activity from ictal ElectroEncephaloGram (EEG) data is an essential preprocessing step in diagnosis and study of epileptic disorders. Indeed, at the very beginning of seizures, ictal EEG has a low amplitude and its morphology in the time domain is quite similar to muscular activity. Contrary to the time domain, ictal signals have specific characteristics in the time-frequency domain. In this paper, we use the time-frequency signature of ictal discharges as a priori information on the sources of interest. To extract the time-frequency signature of ictal sources, we use the Canonical Correlation Analysis (CCA) method. Then we propose two time-frequency based semi-blind source separation approaches, namely the Time-Frequency-Generalized Eigen Value Decomposition (TF-GEVD) and the Time-Frequency-Denoising Source Separation (TF-DSS), for the denoising of ictal signals based on these time-frequency signatures. The performance of the proposed methods is compared with that of CCA and Independent Component Analysis (ICA) approaches for the denoising of simulated ictal EEGs and of real ictal data. The results show the superiority of the proposed methods in comparison with CCA and ICA.
\end{abstract}

Index Terms-Generalized EigenValue Decomposition (GEVD), Denoising Source Separation (DSS), Canonical Correlation Analysis (CCA), Semi-blind source separation, ElectroEncephaloGram (EEG), fast ictal activity, epileptic seizure

\section{INTRODUCTION}

$\mathbf{E}$ PILEPSY is one of the most common neurological disorders. In diagnosis and study of epileptic disorders, ElectroEncephaloGraphy (EEG) is a widely used technology. In particular, the recording and interpretation of EEG ictal discharges during seizures in patients with refractory partial epilepsy is a key component in the presurgical evaluation of these patients that contributes, along with other morphological and functional explorations, to the delineation of the epileptogenic zone. The intracerebral recording of these discharges in partial epilepsies have shown the occurrence of low voltage fast activity at the beginning of seizures. These rapid ictal discharges are considered as electrophysiological signatures of the epileptogenic zone, i.e. of the brain region(s) from where the seizure starts [3], [4], [21]. The surgical resection of regions generating rapid ictal discharges has been correlated with a good surgical outcome [1], [5], [22]. Therefore, tracking these rapid discharges on scalp EEG is critical for the presurgical evaluation of patients who are candidates for selective surgery. Unfortunately, the rapid ictal discharges are of low amplitude and therefore difficult to detect on scalp EEG, even more so when signals are contaminated with various kind of artifacts. Among them, muscular activity arising from the contraction of head muscles is often associated with clinical symptoms occurring at the onset of seizures, and is particularly difficult to remove due to its broad distribution in frequency and its unstereotyped spatial distribution.

Different denoising methods have been proposed and tested to denoise ictal signals, from simple low-pass filtering to more complicated algorithms, such as matched filtering [16] and Blind Source Separation (BSS) methods. Principal Component Analysis (PCA), Independent Component Analysis (ICA) and Canonical Correlation Analysis (CCA) are the most common BSS techniques. These techniques have been used to denoise ictal EEG data [10], [12], [14], [17]-[19] and were shown to be efficient to retrieve the EEG rhythmic activity during seizures. However, these studies have not specifically address the difficult issue of denoising fast ictal activity arising at the onset of seizures. As their names indicate, the BSS methods do not use any prior information from subspace of interest. But in some applications, useful knowledge about sources of interest is either available or can be achieved. In our application, although ictal fast activity does not have a specific morphology in the time domain, its signature in the time-frequency domain is narrow-band while that of muscle activity is broad-band [14]. These characteristics can be used to separate ictal fast activity from muscular activity.

In this paper, we propose two methods for denoising ictal EEG data based on the Generalized EigenValue Decomposition (GEVD) and on the Denoising Source Separation (DSS) [15] frameworks, respectively. Indeed, we are interested in proposing semi-blind source separation methods instead of completely blind ones. To this end, we look for some a priori information on sources of interest to define the covariance matrix in the GEVD method and in the denoising step of the DSS method. Accordingly, the proposed semi-blind methods, namely TF-GEVD and TF-DSS, use the time-frequency signature of ictal discharges to denoise ictal EEGs. To extract the time-frequency information on ictal sources, we use the 
CCA approach that has been shown to be the most efficient method to date for denoising ictal EEG signals contaminated by muscular activity [10], [12], [14]. In the rest of this paper, we first present the problem formulation. Section III begins with the description of the CCA-based method used to extract the time-frequency signature of ictal subspace. Then we define the general formulation of the proposed semi-blind methods and mathematically compare their formulations. In section IV, we compare the performance of the proposed algorithms with that of CCA [10] and ICA [7] for the denoising of both simulated and real ictal data. This section is followed by the conclusion.

\section{PROBLEM FORMULATION AND NOTATIONS}

The ictal EEG data is recorded by means of $M$ electrodes placed over the scalp according to one of the international systems. Mean subtraction is then performed. That is, for each channel, the mean of the corresponding time series is subtracted from each value in order to remove constant artifacts, leaving $M$ EEG signals oscillating positively and negatively around the zero line. The $N$ samples of the resulting $M$-dimensional centered ictal EEG signal can then be modeled as follows [2]:

$\boldsymbol{x}[n]=\boldsymbol{A}^{(e)} \boldsymbol{s}^{(e)}[n]+\boldsymbol{A}^{(b)} \boldsymbol{s}^{(b)}[n]+\boldsymbol{A}^{(m)} \boldsymbol{s}^{(m)}[n]+\boldsymbol{\nu}[n]$

where $\left\{\boldsymbol{s}^{(e)}[n]\right\},\left\{\boldsymbol{s}^{(b)}[n]\right\},\left\{\boldsymbol{s}^{(m)}[n]\right\}$ and $\{\boldsymbol{\nu}[n]\}$ are centered vector signals representing $P_{e}$ ictal epileptic activities, $P_{b}$ background activities, $P_{m}$ muscular activities and an $M$ dimensional instrument noise, respectively, with $P_{e}+P_{b}+$ $P_{m}>M$. The mixing matrices $\boldsymbol{A}^{(e)}, \boldsymbol{A}^{(b)}$ and $\boldsymbol{A}^{(m)}$ of size $\left(M \times P_{e}\right),\left(M \times P_{b}\right)$ and $\left(M \times P_{m}\right)$, model the transfer function from all possible activities within the brain to scalp electrodes.

In addition, we can assume that the four vector signals $\left\{\boldsymbol{s}^{(e)}[n]\right\},\left\{\boldsymbol{s}^{(b)}[n]\right\},\left\{\boldsymbol{s}^{(m)}[n]\right\}$ and $\{\boldsymbol{\nu}[n]\}$ generate four linearly independent subspaces of $\mathbb{R}^{N}$, respectively, since they correspond to different physiological/physical phenomena. Let $\tilde{P}_{e}, \tilde{P}_{b}, \tilde{P}_{m}$ and $\tilde{P}_{\nu}$ be the dimensions of the four previous subspaces. Then, we will also assume the following inequality $\tilde{P} \leq M$ where $\tilde{P}=\tilde{P}_{e}+\tilde{P}_{b}+\tilde{P}_{m}+\tilde{P}_{\nu}$.

As a result, at best we can hope to identify the four subspaces generated by the epileptic activities, the muscular activities, the background activities and the instrument noise, respectively, but not exactly the $P=P_{e}+P_{b}+P_{m}$ electrophysiological activities and the $M$ noise signals involved in (1). Note that this subspace identification is sufficient for the EEG denoising problem since we do not want to exactly extract the $P_{e}$ epileptic activities; in fact, we just want to remove the contribution of the muscular activities, the background activities and the instrument noise from the scalp data $\{\boldsymbol{x}[n]\}$.

Let the $\tilde{P}_{e}$-dimensional signal $\left\{\tilde{\boldsymbol{s}}^{(e)}[n]\right\}$, the $\tilde{P}_{b}$-dimensional signal $\left\{\tilde{\boldsymbol{s}}^{(b)}[n]\right\}$, the $\tilde{P}_{m}$-dimensional signal $\left\{\tilde{\boldsymbol{s}}^{(m)}[n]\right\}$ and the $\tilde{P}_{\nu}$-dimensional signal $\left\{\tilde{\boldsymbol{s}}^{(\nu)}[n]\right\}$ be an orthogonal centered vector basis of the epileptic, background, muscular and instrument noise subspaces, respectively, and be mutually orthogonal. Then there exists four matrices of coordinates, namely $\tilde{\boldsymbol{A}}^{(e)}, \tilde{\boldsymbol{A}}^{(b)}, \tilde{\boldsymbol{A}}^{(m)}$ and $\tilde{\boldsymbol{A}}^{(\nu)}$, such that:

$$
\begin{aligned}
\boldsymbol{x}[n] & =\tilde{\boldsymbol{A}}^{(e)} \tilde{\boldsymbol{s}}^{(e)}[n]+\tilde{\boldsymbol{A}}^{(b)} \tilde{\boldsymbol{s}}^{(b)}[n]+\tilde{\boldsymbol{A}}^{(m)} \tilde{\boldsymbol{s}}^{(m)}[n]+\tilde{\boldsymbol{A}}^{(\nu)} \tilde{\boldsymbol{s}}^{(\nu)}[n] \\
& =\tilde{\boldsymbol{A}} \tilde{\boldsymbol{s}}[n]
\end{aligned}
$$

where the $\tilde{P}$ rows $\left\{\tilde{s}_{p}[n]\right\}$ of the vector signal $\{\tilde{\boldsymbol{s}}[n]\}$ are pairwise orthogonal. Consequently, by assigning the following inner product:

$$
\begin{aligned}
& \forall\{y[n]\} \in \mathbb{R}^{N}, \quad \forall\{z[n]\} \in \mathbb{R}^{N}, \\
&\langle\{y[n]\},\{z[n]\}\rangle=\sum_{n=1}^{N} y[n] z[n]
\end{aligned}
$$

to $\mathbb{R}^{N}$, the $(\tilde{P} \times \tilde{P})$ matrix $\boldsymbol{C}_{\tilde{\boldsymbol{s}}}$, whose $\left(p_{1}, p_{2}\right)$-th component is equal to $\left\langle\left\{\tilde{s}_{p_{1}}[n]\right\},\left\{\tilde{s}_{p_{2}}[n]\right\}\right\rangle$, is a diagonal matrix.

As a result, by computing an orthogonal vector basis $\left\{\tilde{\boldsymbol{s}}^{(e)}[n]\right\}$ of the epileptic subspace and the corresponding $\tilde{\boldsymbol{A}}^{(e)}$ matrix of coordinates from the measurements $\{\boldsymbol{x}[n]\}$, the $M$ dimensional signal $\left\{\boldsymbol{x}^{(e)}[n]\right\}$ given by $\boldsymbol{x}^{(e)}[n]=\tilde{\boldsymbol{A}}^{(e)} \tilde{\boldsymbol{s}}^{(e)}[n]$ will represent the denoised ictal EEG data.

In the following sections, the $p$-th ictal source and the $p$ th source will correspond to the $p$-th vector of the epileptic basis $\left\{\tilde{\boldsymbol{s}}^{(e)}[n]\right\}$ and the $p$-th vector of the whole basis $\{\tilde{\boldsymbol{s}}[n]\}$, respectively. In addition, we denote by $T_{x_{m}}[n, k]$ the linear time-frequency transform of the $m$-th recorded ictal EEG signal, in the time sample $n$ and frequency sample $k$. The $M$ dimensional vector $T_{\boldsymbol{x}}[n, k]$ then contains the $(n, k)$-th linear time-frequency samples of the $M$ channels. Therefore, according to (2), for each couple $(n, k)$ of $\{1, \ldots, N\} \times\{1, \ldots, K\}$, we have:

$$
T_{\boldsymbol{x}}[n, k]=\sum_{p=1}^{\tilde{P}} \tilde{\boldsymbol{a}}_{p} T_{\tilde{s}_{p}}[n, k]=\tilde{\boldsymbol{A}} T_{\tilde{\boldsymbol{s}}}[n, k]
$$

where $T_{\tilde{s}_{p}}[n, k], T_{\tilde{s}}[n, k]$ denote the linear time-frequency transform of the $p$-th source and that of the $\tilde{P}$ sources in the time-frequency sample $(n, k)$, respectively.

According to the property that the energy of the $\tilde{P}_{e}$ ictal epileptic activities is local in the time-frequency domain [14], for the $p$-th ictal source we assume that the largest portion of its energy is reserved in a time-frequency subset $T F^{(p)}$ of $\{1, \ldots, N\} \times\{1, \ldots, K\}$. Thus, we aim at computing an orthogonal vector basis of the epileptic subspace using this a priori information on the ictal sources through specific timefrequency matrices. Let us assign the following inner product:

$$
\begin{array}{r}
\forall\{y[n, k]\} \in \mathbb{C}^{N \times K}, \quad \forall\{z[n, k]\} \in \mathbb{C}^{N \times K}, \\
\langle\{y[n, k]\},\{z[n, k]\}\rangle=\sum_{n=1}^{N} \sum_{k=1}^{K} y[n, k] z[n, k]^{*}
\end{array}
$$

to $\mathbb{C}^{N \times K}$, where $z[n, k]^{*}$ denotes the conjugate of $z[n, k]$. Given an $L$-dimensional signal $\{\boldsymbol{u}[n]\}$ and its linear timefrequency transform $\left\{T_{\boldsymbol{u}}[n, k]\right\}$, we define the time-frequency matrices $\widehat{\boldsymbol{C}}_{\boldsymbol{u}}$ and $\widehat{\boldsymbol{C}}_{\boldsymbol{u}}^{(p)}$ as the $(L \times L)$ matrices whose $\left(\ell_{1}, \ell_{2}\right)$-th component is equal to $\left\langle\left\{T_{u_{\ell_{1}}}[n, k]\right\},\left\{T_{u_{\ell_{2}}}[n, k]\right\}\right\rangle$ and $\left\langle\left\{T_{u_{\ell_{1}}}[n, k] \mathbb{1}_{T F^{(p)}}[n, k]\right\},\left\{T_{u_{\ell_{2}}}[n, k] \mathbb{1}_{T F^{(p)}}[n, k]\right\}\right\rangle$, respectively, where $\mathbb{1}_{T F^{(p)}}[n, k]$ is equal to one if $(n, k)$ belongs 


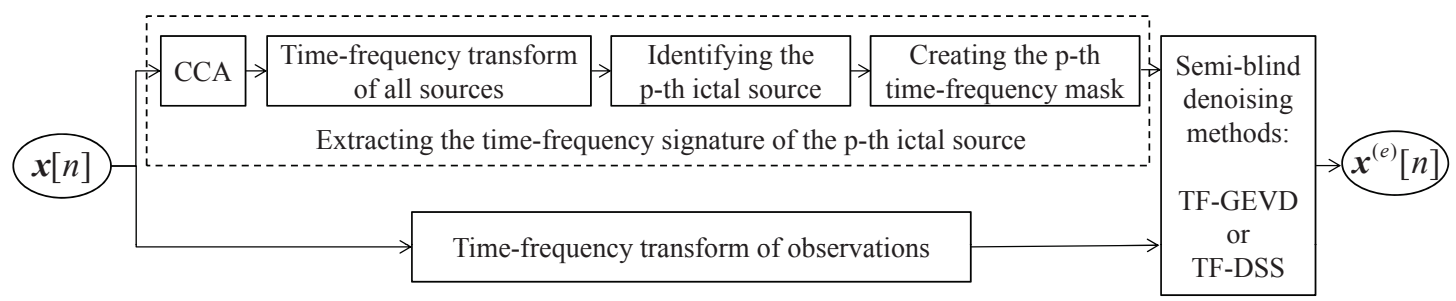

Fig. 1. The flow chart of the denoising procedure to extract the $p$-th ictal subspace corresponding to the $p$-th ictal source.

to $T F^{(p)}$ and zero otherwise. We thus define the $(L \times L)$ timefrequency matrix $\widehat{C}_{\boldsymbol{u}}^{(\bar{p})}$ by $\widehat{\boldsymbol{C}}_{\boldsymbol{u}}^{(\bar{p})}=\widehat{\boldsymbol{C}}_{\boldsymbol{u}}-\widehat{\boldsymbol{C}}_{\boldsymbol{u}}^{(p)}$. Note that for $L=1$, i.e. for a monodimensional signal $\{u[n]\}$, we will replace the bold capital letter $C$ by the lower case letter $c$. It is also noteworthy that $\sharp$ will denote the pseudo-inverse operator in the following sections.

\section{Methodology}

In this section, we propose two semi-blind source separation methods to denoise ictal EEG signals. They use some a priori information on the ictal sources in the time-frequency domain that should be initially extracted. The general flow chart of the proposed methods to extract the ictal subspace corresponding to the $p$-th ictal source is shown in figure 1. As shown in this figure, the proposed methods consist of three main steps: 1) extracting the linear time-frequency signature of the $p$-th ictal source, 2) calculating the time-frequency transform of the observations and 3) applying a time-frequency-based semiblind denoising method to linear time-frequency transform of the observations. The proposed methods only differ in the third block where either TF-GEVD or TF-DSS is used for the denoising procedure. These steps are explained in details in the rest of this section and then the relation between the two proposed methods is studied.

\section{A. Extracting time-frequency mask corresponding to each ictal source}

The CCA approach is a BSS method that extracts the sources from observations with the main assumption that the sources are mutually uncorrelated as well as maximally autocorrelated [10]. As shown in previous studies [10], [14], CCA surpasses other methods for the denoising of ictal signals. Nevertheless, it can not achieve good results in some instances such as low SNRs. In the method we propose, we use the CCA method to extract some a priori information on ictal sources in order to improve the ictal noise cancelation process. To this end, we apply the CCA method on the observed EEGs to obtain $M$ sources. The next step consists in selecting the sources of ictal activity. A visual selection of ictal sources from their time course is difficult and sometimes impossible. Therefore different methods have been proposed to select ictal sources from all extracted ones, such as methods based on the timefrequency transform of each source [14] and methods based on spectral coherence of sources [18]. To select the sources corresponding to ictal activities, we use the linear time-frequency representation of each source and then we choose the sources of interest based on a signature that consists of a narrowband, high frequency mode at the beginning of the seizure, that decreases in frequency over time. Figures 2(a) and 2(b) show an example of the selected ictal source extracted by CCA and its linear time-frequency representation, respectively. In this paper, we use the Continuous Wavelet Transform (CWT) with real Morlet mother wavelet which shows a good representation of ictal sources in the time-frequency domain. As explained later in this section, since the real-valued data should be used in the proposed algorithms, CWT with real Morlet mother wavelet is an appropriate choice. After selecting the sources of interest, we use an automatic procedure to generate the timefrequency mask. To this end, we use an appropriate threshold to select the dominant time-frequency samples from the linear time-frequency representation of each source of interest. The threshold used for the source of interest $\tilde{s}_{p}$ is obtained as $t h_{\tilde{s}_{p}}=m_{\left|T_{\tilde{s}_{p}}\right|}+\sigma_{\left|T_{\tilde{s}_{p}}\right|}$ where $m_{\left|T_{\tilde{s}_{p}}\right|}$ and $\sigma_{\left|T_{\tilde{s}_{p}}\right|}$ are the mean value and standard deviation of the absolute values of $T_{\tilde{s}_{p}}[n, k]$ over all $(n, k) \in\{1, \ldots, N\} \times\{1, \ldots, K\}$, respectively. Then the absolute value of $T_{\tilde{s}_{p}}[n, k]$ is compared with $t h_{\tilde{s}_{p}}$ at each timefrequency sample $(n, k)$ and the $p$-th time-frequency mask $S^{(p)}$ is obtained as follows:

$$
S^{(p)}[n, k]=\left\{\begin{array}{cc}
1 & \text { if }\left|T_{\tilde{s}_{p}}[n, k]\right| \geq t h_{\tilde{s}_{p}} \\
0 & \text { o.w. }
\end{array}\right.
$$

Then, we consider the obtained time-frequency mask $S^{(p)}$ as a binary image and modify it by using morphological operators. To this end, we use the morphological "closing" operator [11] with a disk structuring element of size 1 . The "closing" operator performs morphological closing on the grayscale or binary image by applying a dilation followed by an erosion, using the same structuring element for both operations. By using this operator, the holes in the initial time-frequency mask are filled. Figure 2(c) shows the timefrequency mask created by the above-mentioned procedure. The time-frequency samples corresponding to the $p$-th ictal source are then reserved in the set $T F^{(p)}$ :

$$
T F^{(p)}=\left\{\left(n^{(p)}, k^{(p)}\right) \in \text { the } p \text {-th ictal mask }\right\}
$$

The obtained samples will then be used in the proposed TFGEVD or TF-DSS methods to denoise ictal signals.

\section{B. $T F-G E V D$}

In this section, we propose a Generalized EigenValue Decomposition-based method to identify the $p$-th ictal source 

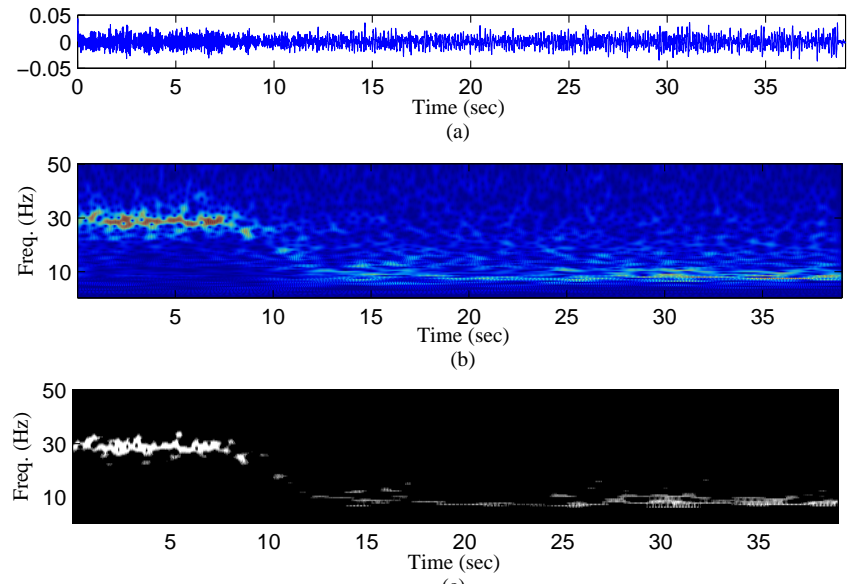

(c)

Fig. 2. Procedure used to create the time-frequency mask corresponding to an ictal source, (a) selected ictal source generated by CCA, (b) timefrequency representation (absolute value of CWT) of the ictal source and (c) time-frequency mask corresponding to the ictal source of (a).

using its time-frequency support $T F^{(p)}$. More particularly, we aim at computing the $M$-dimensional vector $\boldsymbol{w}_{p}$ such that the signal $\left\{y_{p}^{(e)}[n]\right\}$ defined by $y_{p}^{(e)}[n]=\boldsymbol{w}_{p}^{\top} \boldsymbol{x}[n]$ is an estimate of the $p$-th ictal source. To this end, we maximize the following quotient of energies on and outside the time-frequency support $T F^{(p)}$ :

$$
\boldsymbol{J}^{(p)}(\boldsymbol{w})=\frac{\widehat{c}_{y_{p}^{(e)}}^{(p)}}{\widehat{c}_{y_{p}^{(e)}}^{(\bar{p})}}=\frac{\boldsymbol{w}^{\top} \widehat{\boldsymbol{C}}_{\boldsymbol{x}}^{(p)} \boldsymbol{w}}{\boldsymbol{w}^{\top} \widehat{\boldsymbol{C}}_{\boldsymbol{x}}^{(\bar{p})} \boldsymbol{w}}
$$

which is a Rayleigh quotient. Finding the argument $\boldsymbol{w}_{p}$ of the maximum of (8) is equivalent to solving a specific GEVD problem, i.e. computing the eigenvector associated with the largest eigenvalue of the $(M \times M)$ matrix $\left(\widehat{\boldsymbol{C}}_{\boldsymbol{x}}^{(\bar{p})}\right)^{-1} \widehat{\boldsymbol{C}}_{\boldsymbol{x}}^{(p)}$.

By executing the above-mentioned procedure for each $p \in$ $\left\{1, \ldots, \tilde{P}_{e}\right\}, \tilde{P}_{e}$ vectors $\boldsymbol{w}_{p}$ are extracted and the matrix $\tilde{\boldsymbol{A}}^{(e)}$ of coordinates can be estimated as $\boldsymbol{X}\left(\boldsymbol{Y}^{(e)}\right)^{\sharp}$ where $\boldsymbol{X}$ and $\boldsymbol{Y}^{(e)}$ are the $(M \times N)$ and $\left(\tilde{P}_{e} \times N\right)$ matrices standing for the $M$-dimensional signal $\{\boldsymbol{x}[n]\}$ and the $\tilde{P}_{e}$-dimensional signal $\left\{\boldsymbol{y}^{(e)}[n]\right\}$, respectively, with $\boldsymbol{y}^{(e)}[n]=\left[y_{1}^{(e)}[n], \cdots, y_{\tilde{P}_{e}}^{(e)}[n]\right]^{\top}$.

\section{C. $T F-D S S$}

The DSS [15] method is a relatively recent framework which can be used to design new source separation algorithms. This framework can be optimized to generate a wide range of source separation algorithms, from completely to partially blind methods to solve specific problems. In this framework, the source separation algorithms incorporate denoising methods, such that various kinds of prior knowledge are formulated based on denoising goals [15]. In this section, we propose a DSS-based method to denoise ictal EEG data in the timefrequency domain.

The TF-DSS algorithm is based on a whitening of the linear time-frequency transform of observations, $\left\{T_{\boldsymbol{x}}[n, k]\right\}$, such that the time-frequency matrix $\widehat{C}_{z}$ of the output signal
$\boldsymbol{z}[n]=\boldsymbol{\Theta}^{\sharp} \boldsymbol{x}[n]$ is equal to the identity matrix. In practice, $\boldsymbol{\Theta}$ is computed as a square root of the time-frequency matrix $\widehat{C}_{x}$. By means of this whitening procedure, the matrix $\tilde{\boldsymbol{A}}$ defined in (2) is transformed into an orthogonal matrix of size $(\tilde{P} \times \tilde{P})$.

The four consequent steps are the main steps of the TF-DSS method which are repeated in an iterative procedure to extract the $p$-th ictal source [15]. First, by using a $\tilde{P}$-dimensional initial vector $\boldsymbol{w}_{p}$ of unit norm, a noisy estimate of the timefrequency transform of the $p$-th ictal source for each timefrequency sample $(n, k)$ is calculated as follows:

$$
T_{y_{p}^{(e)}}[n, k]=\boldsymbol{w}_{p}^{\top} T_{\boldsymbol{z}}[n, k]
$$

The next step, called the denoising step, is the main stage of the algorithm. In this stage, the current estimated timefrequency transform of the $p$-th ictal source, for each timefrequency sample $(n, k)$, is modified or denoised as follows:

$$
T_{y_{p}^{(e)}}^{+}[n, k]=T_{y_{p}^{(e)}}[n, k] \mathbb{1}_{T F^{(p)}}[n, k]
$$

In the third and fourth steps, by using the denoised time-frequency transform of the $p$-th ictal source, namely $\left\{T_{y_{p}^{(e)}}^{+}[n, k]\right\}$, a new estimation of the mixing vector is calculated and normalized as follows:

$$
\begin{aligned}
& \boldsymbol{w}_{p}^{+}= {\left[w_{p, 1}^{+}, \cdots, w_{p, \tilde{P}_{e}}^{+}\right]^{\top} \quad \text { with } } \\
& w_{p, p^{\prime}}^{+}=\left\langle\left\{T_{z_{p^{\prime}}}[n, k]\right\},\left\{T_{y_{p}^{(e)}}^{+}[n, k]\right\}\right\rangle \\
& \boldsymbol{w}_{p}=\frac{\boldsymbol{w}_{p}^{+}}{\left\|\boldsymbol{w}_{p}^{+}\right\|}
\end{aligned}
$$

Then, the present estimated mixing vector $\boldsymbol{w}_{p}$ is passed to the first step given by (9) and this procedure continues until its convergence. The global convergence of the iterative DSS scheme is proved in [15]. The outputs of the algorithm, $\boldsymbol{w}_{p}$ and the signal $\left\{y_{p}^{(e)}[n]\right\}$, which is defined by $y_{p}^{(e)}[n]=\boldsymbol{w}_{p}^{\top} \boldsymbol{z}[n]$, are the separator and an estimate of the $p$-th ictal source.

It should be noted that if we have extracted the first $p-1$ sources, to extract the $p$-th source, the fourth step given by (12) can be replaced by:

$$
\begin{aligned}
\boldsymbol{w}_{p}^{\perp} & =\boldsymbol{\Pi}^{\perp} \boldsymbol{w}_{p}^{+} \\
\boldsymbol{w}_{p} & =\frac{\boldsymbol{w}_{p}^{\perp}}{\left\|\boldsymbol{w}_{p}^{\perp}\right\|}
\end{aligned}
$$

where the orthogonal projector $\Pi^{\perp}$ is defined by:

$$
\begin{aligned}
\boldsymbol{\Pi}^{\perp} & =\boldsymbol{I}-\boldsymbol{B}^{(p-1)}\left(\boldsymbol{B}^{(p-1)^{\top}} \boldsymbol{B}^{(p-1)}\right)^{-1} \boldsymbol{B}^{(p-1)^{\top}} \\
& =\boldsymbol{I}-\boldsymbol{B}^{(p-1)} \boldsymbol{B}^{(p-1)^{\top}}
\end{aligned}
$$

with:

$$
\boldsymbol{B}^{(p-1)}=\left[\boldsymbol{w}_{1}, \ldots, \boldsymbol{w}_{p-1}\right]
$$

By adding this deflation step to the DSS framework, the convergence to previously extracted ictal sources is prevented [15].

By executing the above-mentioned procedure for each $p \in\left\{1, \ldots, \tilde{P}_{e}\right\}$, all separators $\boldsymbol{w}_{p}$ and consequently the corresponding ictal source are estimated. The matrix $\tilde{\boldsymbol{A}}^{(e)}$ of coordinates can then be obtained similarly to the TF-GEVD method as described at the end of section III-B. 


\section{Relation between TF-GEVD and TF-DSS methods}

In this section, we study the similarity between both proposed algorithms to extract the $p$-th ictal source. First, for the TF-DSS method, we do not consider the deflation step (13), which means that only one ictal source has to be estimated.

In the TF-GEVD method, the GEVD problem of the pair of matrices $\widehat{\boldsymbol{C}}_{\boldsymbol{x}}^{(p)}$ and $\widehat{\boldsymbol{C}}_{\boldsymbol{x}}^{(\bar{p})}$ can be reformulated as an exact joint diagonalization by congruence problem of these two matrices.

On the other hand, when the denoising stage in DSS algorithm is linear, the whole iterative algorithm can be expressed as a closed-form solution. In section III-C, by inserting (9) and (10) into (11), we can show that the four steps of the TF-DSS algorithm can be summarized by the following procedure:

$$
\begin{aligned}
\boldsymbol{w}_{p}^{+} & =\widehat{\boldsymbol{C}}_{\boldsymbol{z}}^{(p)} \boldsymbol{w}_{p} \\
\boldsymbol{w}_{p} & =\frac{\boldsymbol{w}_{p}^{+}}{\left\|\boldsymbol{w}_{p}^{+}\right\|}
\end{aligned}
$$

which is actually the classical power method applied to matrix $\widehat{\boldsymbol{C}}_{\boldsymbol{z}}^{(p)}$ in order to compute its dominant eigenvector $\boldsymbol{w}_{p}$. Let $\boldsymbol{W}^{(p)}$ be the orthogonal matrix of the $\tilde{P}_{e}$ eigenvectors (including $\boldsymbol{w}_{p}$ ) of the symmetric matrix $\widehat{\boldsymbol{C}}_{\boldsymbol{z}}^{(p)}$. Consequently, the $\left(\tilde{P}_{e} \times M\right)$ matrix $\boldsymbol{W}_{\mathrm{DSS}}^{(p)^{\top}}$ given by $\boldsymbol{W}_{\mathrm{DSS}}^{(p)^{\top}}=\boldsymbol{W}^{(p)^{\top}} \boldsymbol{\Theta}^{\sharp}$ diagonalizes by congruence the time-frequency matrix $\widehat{C}_{\boldsymbol{x}}^{(p)}$. Moreover, due to the orthogonality of $\boldsymbol{W}^{(p)}$ and since $\Theta$ is a root square of $\widehat{\boldsymbol{C}}_{\boldsymbol{x}}$, we can easily show that the matrix $\boldsymbol{W}_{\mathrm{DSS}}^{(p)^{\top}}$ also diagonalizes by congruence the time-frequency matrix $\widehat{\boldsymbol{C}}_{\boldsymbol{x}}$. As a result, the TF-DSS algorithm estimates the $p$-th ictal source by means of a joint diagonalization by congruence of the matrices $\widehat{\boldsymbol{C}}_{\boldsymbol{x}}^{(p)}$ and $\widehat{\boldsymbol{C}}_{\boldsymbol{x}}$.

Now by comparing the two joint diagonalization problems corresponding to the TF-GEVD and TF-DSS methods, the former simultaneously diagonalizes the matrices $\widehat{\boldsymbol{C}}_{\boldsymbol{x}}^{(p)}$ and $\widehat{\boldsymbol{C}}_{\boldsymbol{x}}^{(\bar{p})}$, while the latter jointly diagonalizes the matrices $\widehat{\boldsymbol{C}}_{\boldsymbol{x}}^{(p)}$ and $\widehat{\boldsymbol{C}}_{\boldsymbol{x}}$, where $\widehat{\boldsymbol{C}}_{\boldsymbol{x}}=\widehat{\boldsymbol{C}}_{\boldsymbol{x}}^{(p)}+\widehat{\boldsymbol{C}}_{\boldsymbol{x}}^{(\bar{p})}$ according to the definitions given in section II.

Now, if we consider the deflation step (13) for the TF-DSS method, equations (17) and (18) will be changed to:

$$
\begin{aligned}
\boldsymbol{w}_{p}^{\perp} & =\boldsymbol{\Pi}^{\perp} \widehat{\boldsymbol{C}}_{\boldsymbol{z}}^{(p)} \boldsymbol{w}_{p} \\
\boldsymbol{w}_{p} & =\frac{\boldsymbol{w}_{p}^{\perp}}{\left\|\boldsymbol{w}_{p}^{\perp}\right\|}
\end{aligned}
$$

which results in diagonalization of matrix $\boldsymbol{\Pi}^{\perp} \widehat{\boldsymbol{C}}_{\boldsymbol{z}}^{(p)}$. Consequently, the TF-DSS method to extract the $p$-th ictal source can be described as two serial diagonalization steps, say diagonalization of $\widehat{\boldsymbol{C}}_{\boldsymbol{x}}$ and diagonalization of $\boldsymbol{\Pi}^{\perp} \widehat{\boldsymbol{C}}_{\boldsymbol{z}}^{(p)}$. But contrarily to the previous case, it seems that these two diagonalizations cannot be simplified to a joint diagonalization problem preventing us to compare more precisely the TF-DSS and TF-GEVD algorithms when two or more ictal sources have to be estimated.

\section{EXPERIMENTS AND RESULTS}

In this section, we study the effectiveness of the two proposed algorithms to denoise ictal EEG data by using both simulated and real data. We also compare these two algorithms with the CCA and ICA methods. $\mathrm{CoM}_{2}$ was used to represent the ICA approach based on the study proposed in [2] showing the good trade-off between estimation accuracy and numerical complexity of $\mathrm{CoM}_{2}$.

\section{A. Simulated ictal data}

The simulated EEG data were generated using a realistic model developed by our team [8], [9], [20]. To reproduce the complex geometry of the cerebral neocortex, a mesh of the cortical surface at the interface of gray and white matter (BrainVisa, SHFJ, Orsay, France) was built from the segmentation of 3D T1 MRI image. This mesh is composed of 40500 triangles of mean surface $5 \mathrm{~mm}^{2}$. Each triangle of the mesh has been associated with an elementary current dipole located at the centroid of each triangle and perpendicular to its surface. The source of ictal epileptic activity is manually delineated on the mesh. We consider a source in the left superior temporal gyrus consisting of 100 contiguous triangles (patch) which represents a source area around $5 \mathrm{~cm}^{2}$. The temporal dynamics of the activity associated with each triangle are generated by a macroscopic model of neuronal populations [20]. To generate the ictal discharge dynamics, we apply the settings to get a fast activity during the first 10 seconds and then tune the parameters to obtain a slow rhythmic activity during the last 15 seconds. Between these two activities, we consider a linear transition period of $2 \mathrm{~s}$. We assigned this ictal activity to all the dipoles forming the patch. From this setup, the simulated ictal EEG was obtained at the level of 32 scalp electrodes (placed over the scalp according to the international 10-20 and 10-10 systems). In order to solve the forward problem, we used a realistic head model made of three nested homogeneous surfaces shaping the brain, the skull and the scalp and the Boundary Element Method (ASA, ANT, Enschede, Netherlands). Following this procedure, 50 Monte Carlo simulations were generated (i.e. original simulated data). In order to get noisy data, 50 realizations of a signal of noninterest were added to the original simulated signals with a specified Signal-to-Noise Ratio (SNR). Signals of non-interest were composed of muscle activity, background EEG and instrument noise extracted from real 32-channel EEG data.

To analyze the effectiveness of the proposed algorithms to denoise ictal data, we compared them with the CCA and ICA methods by using two criteria. The first criterion is the Relative Root-Mean-Squared Error (RRMSE) [10] given by:

$$
R R M S E=\frac{\sqrt{\frac{1}{M N} \sum_{m=1}^{M} \sum_{n=1}^{N}\left(x_{m}^{(e)}[n]-\hat{x}_{m}^{(e)}[n]\right)^{2}}}{\sqrt{\frac{1}{M N} \sum_{m=1}^{M} \sum_{n=1}^{N}\left(x_{m}^{(e)}[n]\right)^{2}}}
$$

where $x_{m}^{(e)}[n]$ and $\hat{x}_{m}^{(e)}[n]$ are the original and estimated epileptic subspaces on the $m$-th channel, respectively. We also compared source localization results obtained on noise-free, noisy and denoised data by the 4-ExSo-MUSIC algorithm [6] using the Receiver Operating Characteristic (ROC) curve as a performance criterion. This criterion represents the mathematical expectation of the True Positive Fraction (TPF) as a 
Original EEG

(a)

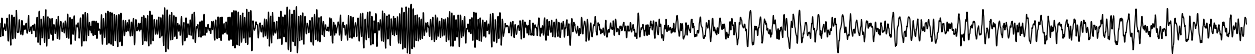

(b)

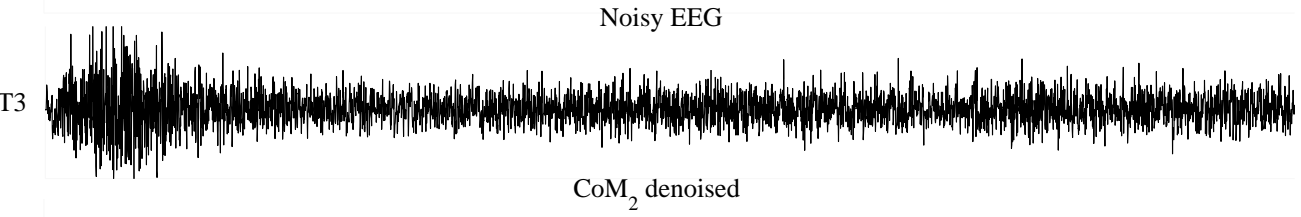

(c)

T3

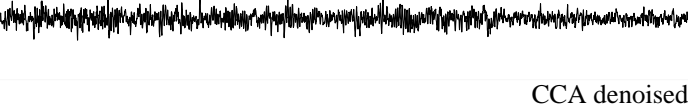

(d)

3

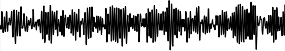

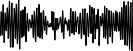

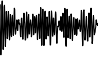

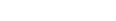

(e)

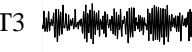

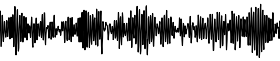

TF-GEVD denoised
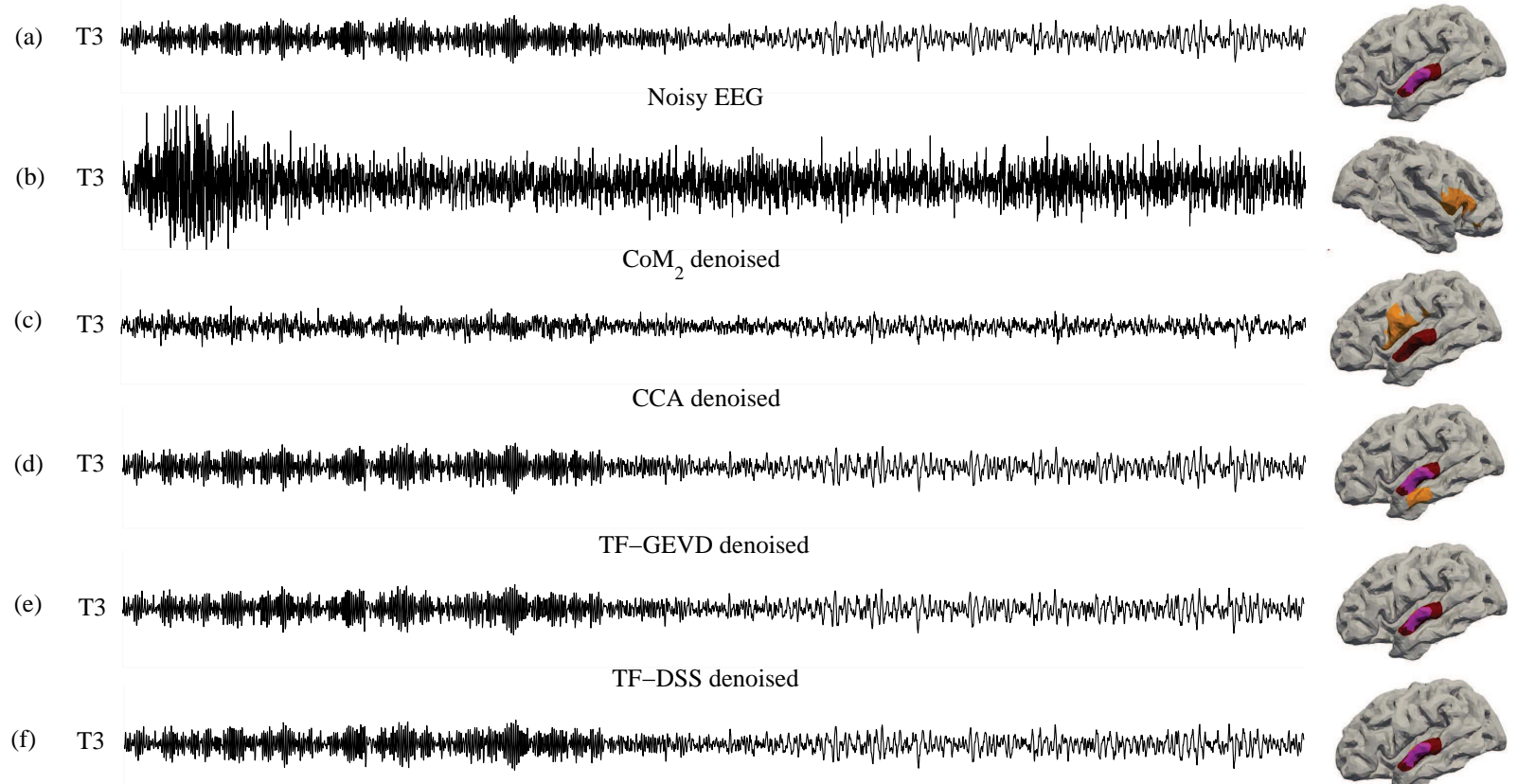

(2)

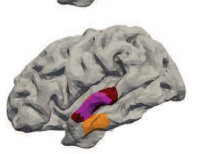

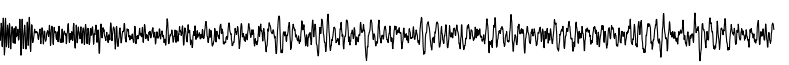

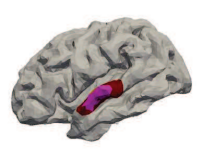

(f)

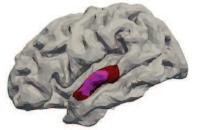

Fig. 3. Denoising results of one trial of simulated ictal data: (a) a noise free ictal EEG, (b) the noisy ictal EEG with the SNR value of $-20 d B$, denoised signal by using (c) $\mathrm{CoM}_{2}$, (d) CCA, (e) TF-GEVD and (f) TF-DSS. For the sake of space, we display the EEG at channel T3 only, as this channel showed the highest amplitude of ictal activity. At the right of each row, the source localization results at the output of 4-ExSo-MUSIC are illustrated for one trial $(\sharp 2)$, as an example. Dark brown: real patch; violet: truly estimated part of the patch; orange: falsely estimated part of the patch.

function of the mathematical expectation of the False Positive Fraction (FPF). The TPF is the fraction between the area of the patch truly retrieved and the total patch area while the FPF is the fraction between the area falsely localized outside the patch and the total cortical area minus the patch area [13].

Figure 3 shows an example of the denoising and source localization results. Figure 3(a) shows the original noise free signal. The maximal amplitude of the simulated ictal signal is obtained at electrode T3. Figure 3(b) illustrates the same trial of ictal data, after adding muscle activity (noisy data: $-20 \mathrm{~dB}$ ). Figures 3(c)-(f) show the denoised signal by using the $\mathrm{CoM}_{2}, \mathrm{CCA}$, TF-GEVD and TF-DSS algorithms, respectively. It should be noted that for the $\mathrm{CoM}_{2}$ algorithm, we varied the number of sources in the range $P \in\{4,8,12,16,20,24,28,32\}$ and chose the value of $P$ yielding the best RRMSE. As shown in these figures, CCA, TF-GEVD and TF-DSS preserved ictal activity at electrode T3. But $\mathrm{CoM}_{2}$ could not perfectly denoise the signal at this electrode and kept some undesired activity at the other electrodes. Although the CCA method worked well to preserve ictal activity in the electrodes that record the highest amplitude of ictal activity in original data, it decreased the activity in other electrodes such as FP1 and FP2. This bias affected the source localization results. The best source localization results are obtained from TF-GEVD and TF-DSS denoised data.

Figure 4 shows the average RRMSE of each one of the four algorithms for different SNR values of -30 to $0 \mathrm{~dB}$. In figure 4(a) and (b), RRMSE is calculated at all channels and at channel T3, respectively. As illustrated in these figures, the errors of the proposed algorithms TF-GEVD and TF-DSS are similar and are clearly smaller than the errors of $\mathrm{CoM}_{2}$ and CCA for low SNR values. These results show that the proposed algorithms are good both on a global point of view (i.e. when all electrodes are considered) and on a more local point of view (i.e. when only T3 electrode is considered).

Figures 5(a)-(c) show the average source localization ROC curves of the four algorithms over the 50 trials for $S N R=$ $-30 d B, S N R=-20 d B$ and $S N R=-10 d B$, respectively. In these figures, the ROC curves corresponding to noisy and noise-free signals are also drawn. The source localization results for a single trial are depicted at the bottom of each column to ease the comparison between the denoising methods. As it can be seen in these figures, the proposed algorithms denoise better, and consequently give better source localization results than those obtained from $\mathrm{CoM}_{2}$ or CCA denoised signals.

\section{B. Real ictal data}

The denoising algorithms were also tested on real ictal data recorded from a 23 years old patient. These data were collected from a long-term video-EEG recording (32 electrodes, 256 $\mathrm{Hz}, \mathrm{BP}$ [0.3-100] Hz) in which we could collect several ictal signals. One of these segments is a low noise ictal EEG with very little muscle activity, that can be used as a reference signal. We apply our proposed algorithms on the signal acquired during a second seizure that was highly contaminated with muscle activity. Six channels of the low noise and noisy ictal signals are shown in figure 6(a) and figure 6(b), respectively. As shown in figure 6(a), the ictal activity of channel $\mathrm{C} 3$ can be segmented to three parts: 1) rhythmic 


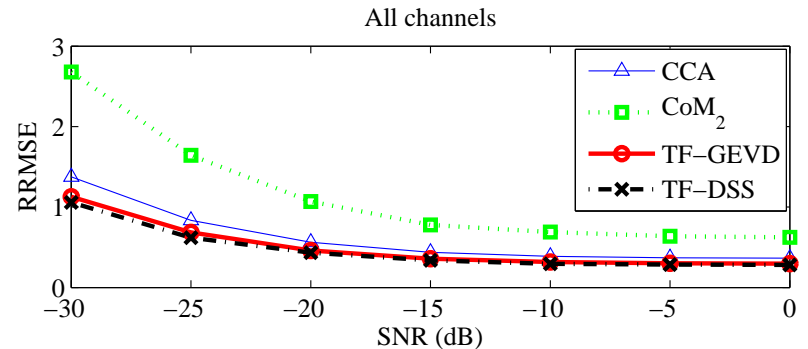

(a)

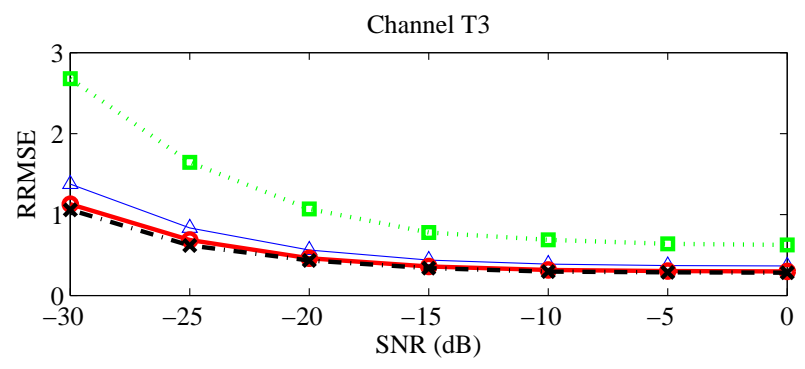

(b)

Fig. 4. The average RRMSE denoising error of each one of the four algorithms in different SNR values of -30 to $0 \mathrm{~dB}$, (a) at all channels and (b) at channel $\mathrm{T} 3$.

spikes (red box), 2) fast activity (green box) and 3) slower irregular activity (blue box). During the second seizure these activities, except the first rhythmic spikes, are covered with muscular activity as shown in figure $6(\mathrm{~b})$.

Figures 7(a) and 7(b) show the time course and timefrequency representations of the channel $\mathrm{C} 3$ for the unnoisy and noisy ictal signals. The ictal fast activity is highlighted in the time-frequency representation of the noise free segment (figure 7(a)). This time-frequency signature is almost invisible in the time-frequency representation of the noisy segment (figure 7(b)). Figures 7(c)-(f) show the time course and timefrequency representations of the channel $\mathrm{C} 3$ of the ictal signals of the second seizure, denoised by the TF-GEVD, TF-DSS, CCA and $\mathrm{CoM}_{2}$ algorithms, respectively. As shown in these figures, the time-frequency signature of the ictal activity appears in the denoised signals. The time course of the denoised signals are cleaner than the noisy data, showing three ictal patterns similar to what had previously been identified during the first unnoisy seizure. Qualitatively, it is noteworthy that although all methods provide fairly good denoising, the best result is obtained with TF-DSS, which could retrieved the fast activity with the highest amplitude. CCA and TF-GEVD gave similar results for the fast activity, but with TF-GEVD some of the spike rhythmic activity has been removed by the denoising procedure. $\mathrm{CoM}_{2}$ can retrieve part of the fast activity but leaves a large amount of muscle activity.

\section{CONCLUSion}

In this paper, we propose two new semi-blind algorithms, namely TF-GEVD and TF-DSS, to denoise ictal EEG data. These methods use the time-frequency signature of ictal sources as a priori information to denoise ictal data. To extract

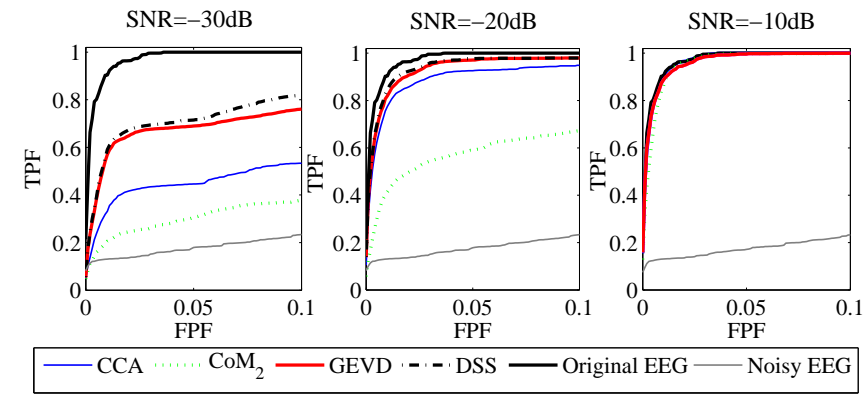

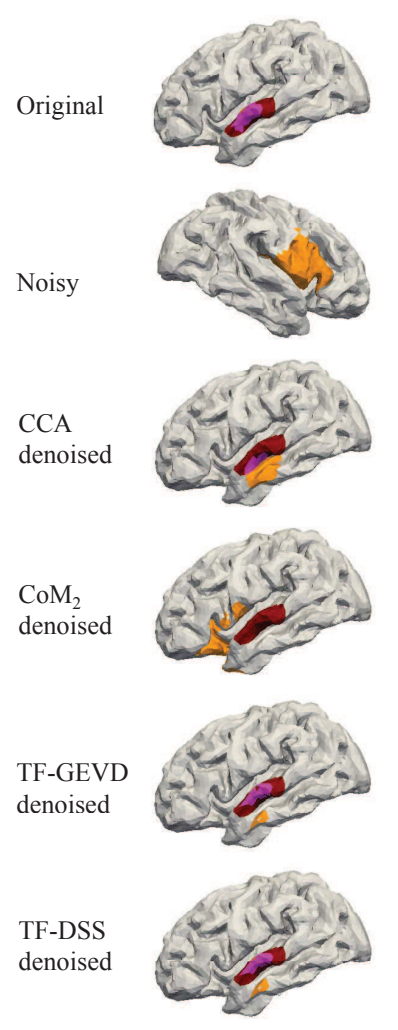

(a)
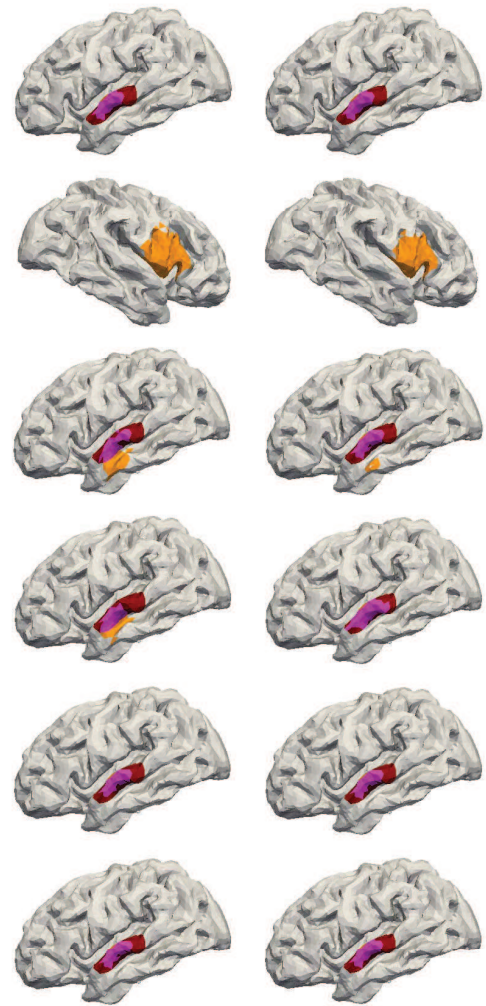

(b)

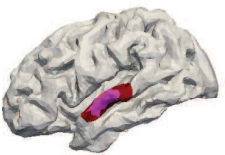

(c)
Fig. 5. Source localization results obtained from original, noisy and denoised ictal simulated data. The average source localization ROC curves (top) of the four algorithms, and the source localization results (bottom) for a single trial $(\sharp 9)$ are displayed for SNR values of (a) $-30 \mathrm{~dB}$, (b) $-20 \mathrm{~dB}$ and (c) -10 dB. Dark brown: real patch; violet: truly estimated part of the patch; orange: falsely estimated part of the patch.

the time-frequency signature of ictal sources, we use a method based on the CCA algorithm. To evaluate and quantify the performance of the proposed methods on denoising of ictal EEGs, we apply them on simulated ictal data and compared their results with those of $\mathrm{CCA}$ and $\mathrm{CoM}_{2}$ algorithms. The results show the superiority of the proposed algorithms in comparison with CCA and $\mathrm{CoM}_{2}$. We also study the utility of the proposed methods in denoising of a real ictal data. In that case, the performance of the denoising algorithms cannot be quantified because the 'ground truth' is unknown. However, a comparison of denoised signals with a different non-noisy ictal signal suggests that the proposed algorithms can be useful for denoising real ictal data. 
Noise free ictal EEG

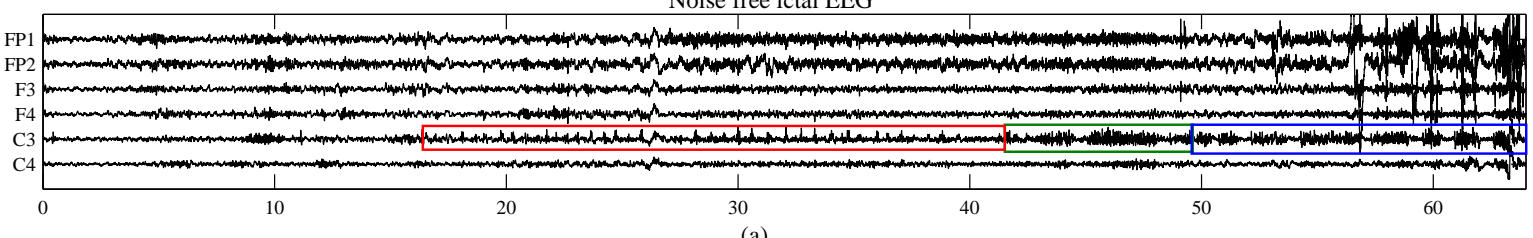

(a)

Noisy ictal EEG

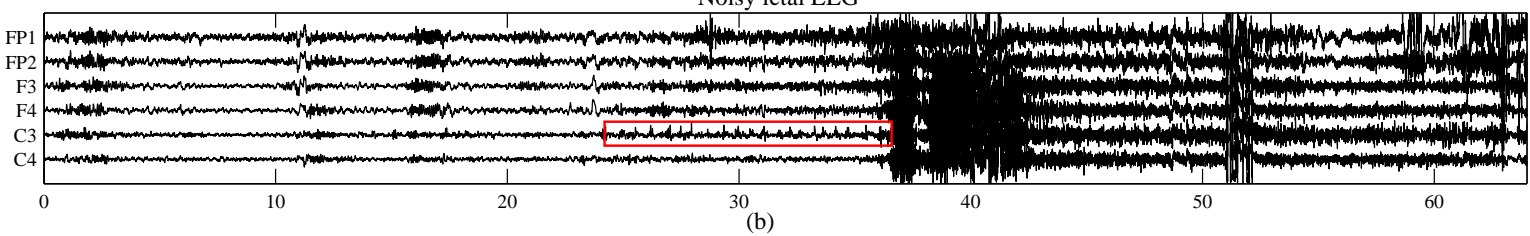

Fig. 6. A real EEG recorded from an epileptic patient during two separate ictal periods: (a) an almost noise free ictal EEG. The channel C3 shows ictal activity consists of three parts: 1) rhythmic spikes (red box), 2) fast activity (green box) and 3) slower irregular activity (blue box). (b) another ictal EEG contaminated by muscle activity.

\section{REFERENCES}

[1] G. Alarcon, C. Binnie, R. Elwes, and C. Polkey, "Power spectrum and intracranial EEG patterns at seizure onset in partial epilepsy," Electroencephalography and clinical neurophysiology, vol. 94, no. 5, pp. 326-337, 1995.

[2] L. Albera, A. Kachenoura, P. Comon, A. Karfoul, F. Wendling, L. Senhadji, and I. Merlet, "ICA-based EEG denoising: a comparative analysis of fifteen methods," Special Issue of the Bulletin of the Polish Academy of Sciences, pp. 407-418, 2012.

[3] J. Bancaud and J. Talairach, La stéréo-électroencéphalographie dans l'épilepsie: informations neurophysiopathologiques apportées par l'investigation fonctionnelle stéreotaxique: rapport a la Société d'électroencephalographie de langue française (Marseille, octobre 1962), 1965

[4] F. Bartolomei, P. Chauvel, and F. Wendling, "Epileptogenicity of brain structures in human temporal lobe epilepsy: a quantified study from intracerebral EEG," Brain, vol. 131, no. 7, pp. 1818-1830, 2008.

[5] F. Bartolomei, D. Cosandier-Rimele, A. McGonigal, S. Aubert, J. Régis, M. Gavaret, F. Wendling, and P. Chauvel, "From mesial temporal lobe to temporoperisylvian seizures: a quantified study of temporal lobe seizure networks," Epilepsia, vol. 51, no. 10, pp. 2147-2158, 2010.

[6] G. Birot, L. Albera, F. Wendling, and I. Merlet, "Localization of extended brain sources from EEG/MEG: The ExSo-MUSIC approach," NeuroImage, vol. 56, no. 1, pp. 102-113, May 2011.

[7] P. Comon, "Independent Component Analysis, a new concept?" Signal Processing, Elsevier, vol. 36, no. 3, pp. 287-314, 1994.

[8] D. Cosandier-Rimélé, J. Badier, P. Chauvel, and F. Wendling, "A physiologically plausible spatio-temporal model for depth-EEG signals recorded with intracerebral electrodes in human partial epilepsy," IEEE Trans. Biomed. Eng., vol. 3, no. 54, pp. 380-388, February 2007.

[9] D. Cosandier-Rimélé, I. Merlet, J. M. Badier, P. Chauvel, and F. Wendling, "The neuronal sources of EEG: Modeling of simultaneous scalp and intracerebral recordings in epilepsy," vol. 42, no. 1, pp. 135146, April 2008

[10] W. De Clercq, A. Vergult, B. Vanrumste, W. Van Paesschen, and S. Van Huffel, "Canonical correlation analysis applied to remove muscle artifacts from the electroencephalogram," IEEE Transactions on Biomedical Engineering, vol. 53, no. 12, pp. 2583-2587, 2006.

[11] R. C. Gonzalez and R. E. Woods, Digital image processing, 2nd. Upper Saddle River, New Jersey 07458: Prentice Hall, 2002.

[12] H. Hallez, M. De Vos, B. Vanrumste, P. Van Hese, S. Assecondi, K. Van Laere, P. Dupont, W. Van Paesschen, S. Van Huffel, and I. Lemahieu, "Removing muscle and eye artifacts using blind source separation techniques in ictal EEG source imaging," Clinical Neurophysiology, vol. 120, no. 7, pp. 1262-1272, 2009.

[13] D. Safieddine, A. Kachenoura, L. Albera, G. Birot, A. Karfoul, A. Pasnicu, A. Biraben, F. Wendling, L. Senhadji, and I. Merlet, "Removal of muscle artifact from EEG data: comparison between stochastic (ICA and CCA) and deterministic (EMD and wavelet-based) approaches," URASIP Journal on Advances in Signal Processing, vol. 127, 2012.
[14] D. Safieddine, "Apport des méthodes statistiques et déterministes à la réduction d'artefacts et de bruits dans les signaux EEG épileptiques," Ph.D. dissertation, Université de Rennes 1, 2012.

[15] J. Sarela and H. Valpola, "Denoising source separation," Journal of Machine Learning Research, vol. 6, pp. 233-272, 2005.

[16] C. Stamoulis and B. S. Chang, "Application of matched-filtering to extract EEG features and decouple signal contributions from multiple seizure foci in brain malformations," in NER'09. 4th International IEEE/EMBS Conference on Neural Engineering, 2009, pp. 514-517.

[17] C. Stamoulis, I. Snchez Fernndez, B. S. Chang, and T. Loddenkemper, "Signal subspace integration for improved seizure localization," in IEEE Annual International Conference of the Engineering in Medicine and Biology Society (EMBC). IEEE, 2012, pp. 1016-1019.

[18] N. P. Subramaniyam, J. Peltola, J. M. Tanskanen, K. Wendel-Mitoraj, J. Hyttinen, and J. Malmivuo, "Source localization based on ictal electroencephalographic recordings," in International Symposium on Biomedical Engineering and Medical Physics. Springer, 2013, pp. 26-30.

[19] E. Urrestarazu, J. Iriarte, M. Alegre, M. Valencia, C. Viteri, and J. Artieda, "Independent component analysis removing artifacts in ictal recordings," Epilepsia, vol. 45, no. 9, pp. 1071-1078, 2004.

[20] F. Wendling, J. J. Bellanger, F. Bartholomei, and P. Chauvel, "Relevance of nonlinear lumped-parameter models in the analysis of depth-EEG epileptic signals," Biol Cybern, vol. 83, pp. 367-78, 2000.

[21] F. Wendling, F. Bartolomei, J.-J. Bellanger, J. Bourien, and P. Chauvel, "Epileptic fast intracerebral EEG activity: evidence for spatial decorrelation at seizure onset," Brain, vol. 126, no. 6, pp. 1449-1459, 2003.

[22] N. M. Wetjen, W. R. Marsh, F. B. Meyer, G. D. Cascino, E. So, J. W. Britton, S. M. Stead, and G. A. Worrell, "Intracranial electroencephalography seizure onset patterns and surgical outcomes in nonlesional extratemporal epilepsy," Journal of neurosurgery, vol. 110, no. 6, p. $1147,2009$. 


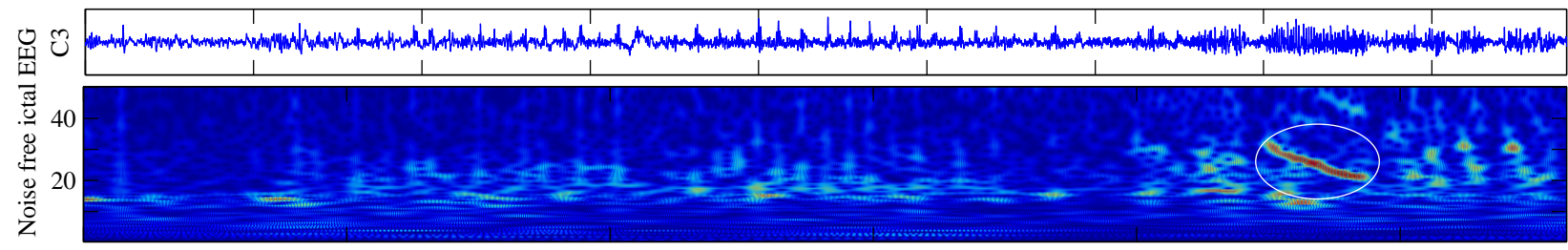

(a)

60

80

100

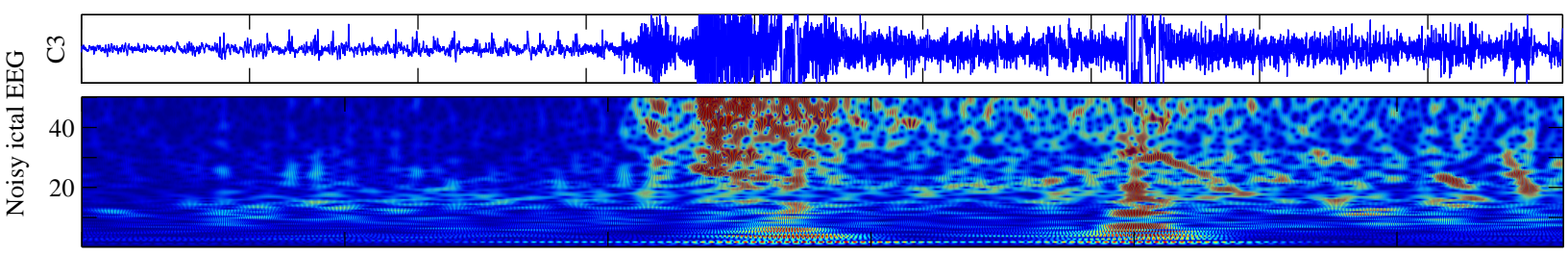

20

(b)

60

80

100
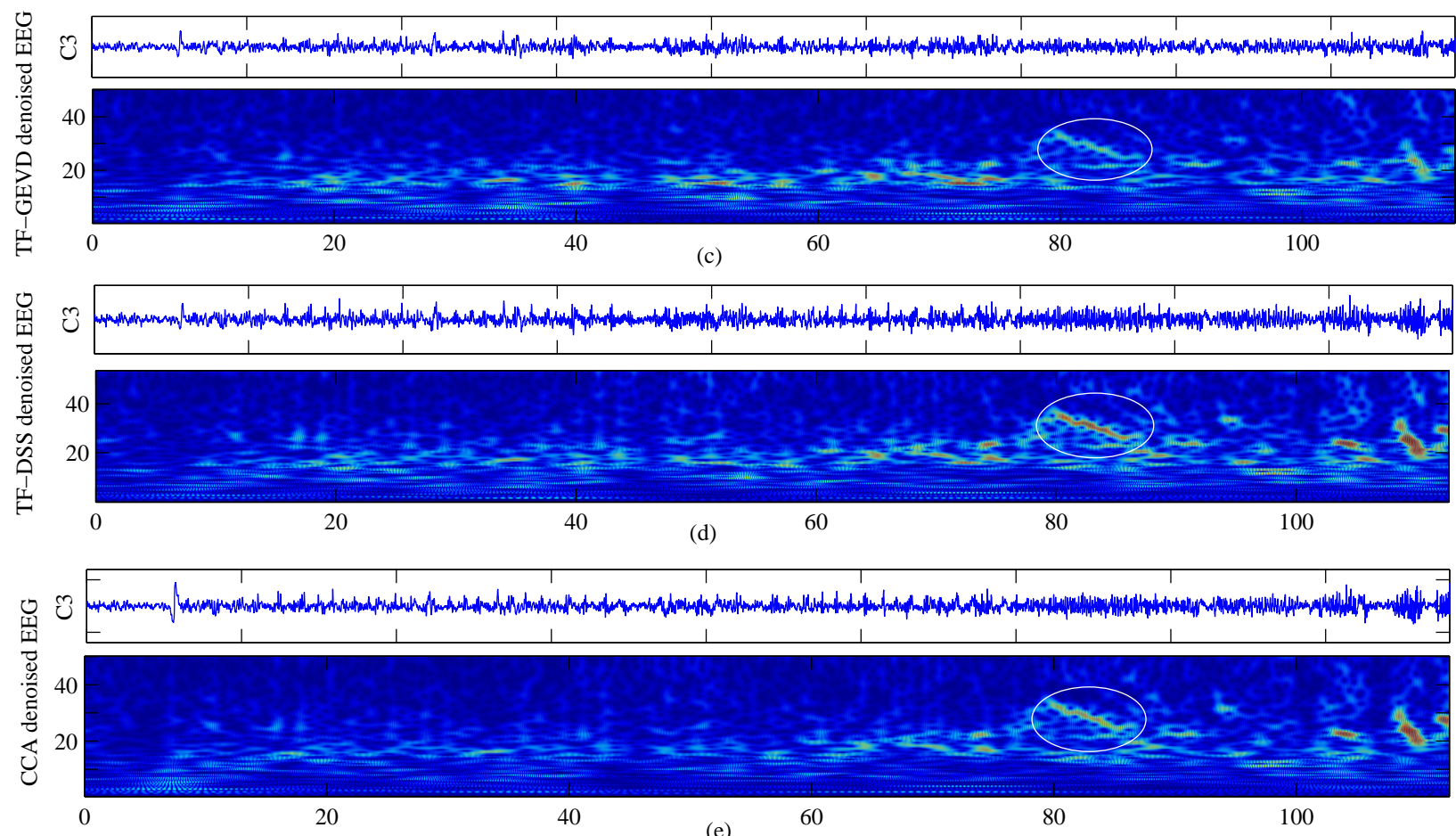

(e)

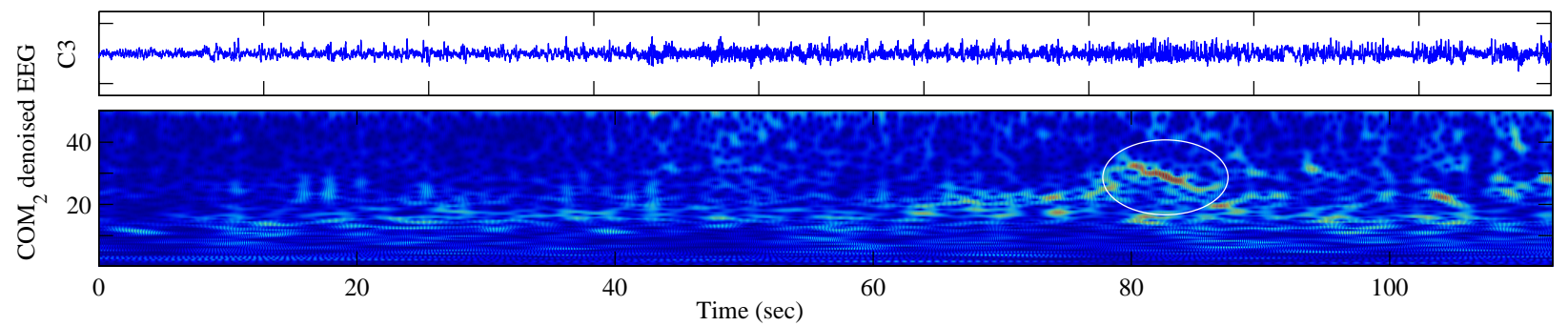

(f)

Fig. 7. The time courses and time-frequency representations at the channel C3 of: (a) the noise free ictal EEG, (b) the noisy ictal segment, (c) TF-GEVD denoised, (d) TF-DSS denoised, (e) CCA denoised and (f) $\mathrm{CoM}_{2}$ denoised EEGs. Ictal fast activity is shown in the white circle on the time-frequency representation. 